\title{
An investigation of the variability of start-up lost times and departure headways at signalized intersections in urban areas
}

\author{
E. Matsoukis \& St. Efstathiadis \\ Transport \& Traffic Engineering Study Unit, \\ Department of Civil Engineering, University of Patras, Greece
}

\begin{abstract}
The number of waiting vehicles that can cross a signalized intersection in a given period of time depends basically on how soon the vehicles begin to move after the signal changes to green and how fast each individual vehicle in the queue reacts to the acceleration of the vehicle immediately ahead. This process continues until all cars in the queue are progressing or have progressed through the intersection. The dissipation of a queue of vehicles after the signal changes to green depends on the reaction time and acceleration characteristics of each individual driver and vehicle. Thus, the total time for a group of vehicles to pass through a signalized intersection can vary considerably depending on the alertness and aggressiveness of the individual drivers, their familiarity with the intersection in question, and the acceleration characteristics of the vehicles, which the drivers control.

This paper attempts to give a new viewpoint on the calculation of the departure headways and of the start-up lost times at signalized intersections in urban areas. Departure headways are the time intervals between vehicles departing from the signalized intersection; start-up lost time is defined as the excess time that is needed for a number of vehicles to pass through the signalized intersection compared with that would be needed if the signal did not exist.

Data was collected for a considerable number of signalized intersection approaches in the Athens, Greece area. Data collection through contemporary techniques included lane width, lane position (inside lane versus outside lane, etc.), time of day (morning-peak versus afternoon-peak) and the posted speed $\operatorname{limit}(48 \mathrm{~km} / \mathrm{h}, 56 \mathrm{~km} / \mathrm{h}, 64 \mathrm{~km} / \mathrm{h}$, or $72 \mathrm{~km} / \mathrm{h})$. Statistical analyses were performed
\end{abstract}


in order to define the elements of the approaches that influence the time headway values.

It was found that the lane width and the lane position (for the ranges that were studied) did not have a significant effect on the lost times and the vehicle headways. The combined effect of time of day and direction had in contrast a significant effect on the lost time and the vehicle headways. The combined effect of the posted speed limit and the area within the city had a significant effect for the lost time when the speed limit was, for example, $45 \mathrm{mph}$ in an open area and for the vehicle headways when the speed limit was $30 \mathrm{mph}$ in a business area.

The final results can be useful for a more effective modeling of the traffic signal calculations performed in all usual traffic engineering techniques.

Keywords: signalized intersections, departure headways, start-up loss time, queue dissipation, urban areas.

\section{Introduction}

The number of waiting vehicles that can cross a signalized intersection in a given period of time depends basically on how soon the vehicles begin to move after the signal changes to green and how fast each individual vehicle in the queue reacts to the acceleration of the vehicle immediately ahead. This process continues until all cars in the queue are progressing or have progressed through the intersection. The dissipation of a queue of vehicles after the signal changes to green depends on the reaction time and acceleration characteristics of each individual driver and vehicle. Thus, the total time for a group of vehicles to pass through a signalized intersection can vary considerably depending on the alertness and aggressiveness of the individual drivers, their familiarity with the intersection in question, and the acceleration characteristics of the vehicles, which the drivers control.

What is accepted is that all drivers, when exposed to the same situation, have different reaction times. That different drivers when placed behind the wheel of identical cars will accelerate from a standing start at different rates is also acknowledged. These characteristics bring in a certain expected variability into any field data collected concerning vehicle performance at intersections.

When the green signal phase begins on an approach to an intersection, stopped vehicles take some time to begin moving; but after a few seconds, the queue discharges at a more or less constant rate termed saturation flow. A basic and well-known model of the variation of queue discharge rate with time in a fully saturated green period is illustrated in Figure 1, [1]. A fully saturated green period is one in which the queue discharge rate remains fairly constant until the green period ends. Saturation flow may vary as a function of items such as layout of the intersection (lane width, grade, etc.), number of turning vehicles, and types of vehicles in the traffic stream, Stokes [2].

Estimation of saturation flow values is of prime importance when determining signalized intersection operation. Saturation flow values are used as input for determining intersection capacity and when using computer models, to simulate and optimize signal systems. 


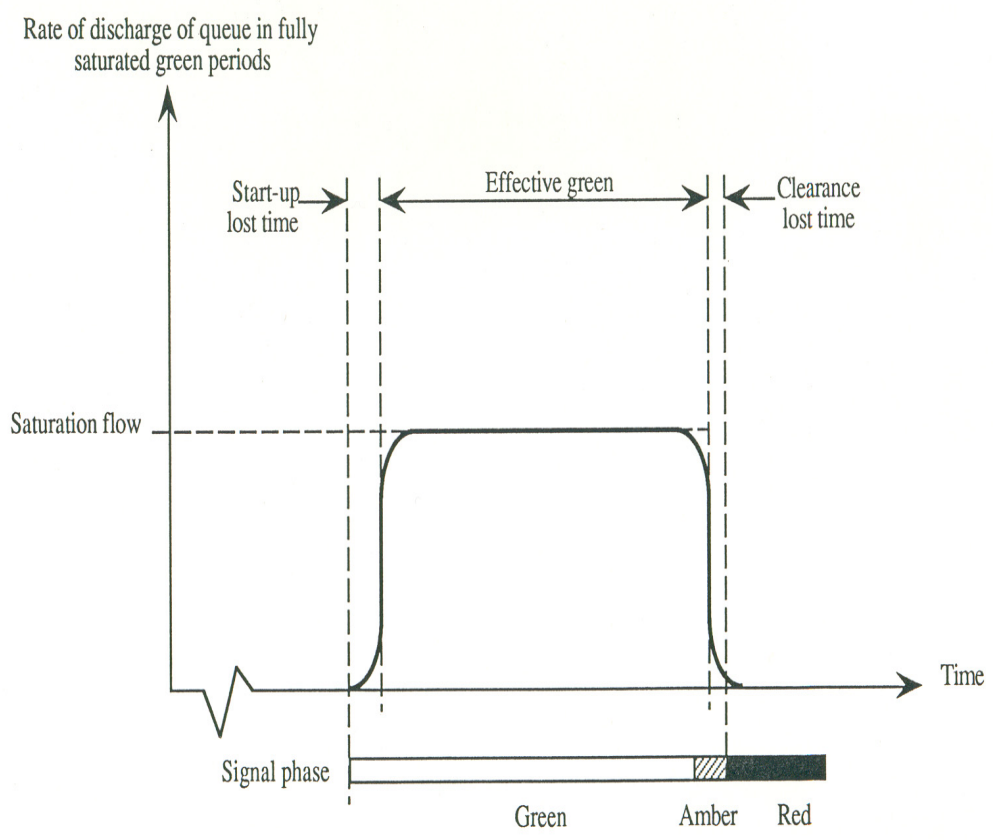

Figure 1: Queue discharge rate in a fully saturated green period.

Two parameters of traffic performance can be used to represent several important characteristics of intersection operation. The two parameters investigated in this study are start-up lost time (or starting delay) and time spacing between vehicles departing from a stopped position to enter a signalized intersection. Information on the variability of these parameters at an intersection and among intersections may have useful applications in studies of intersection capacity and signal timing.

When a traffic signal interrupts the flow of traffic, the vehicles stopped by the signal are delayed during the time the signal is red plus the time required for the vehicles to get started and underway again. This latter delay is commonly called start-up lost time. A generally accepted definition of start-up lost time is the time required for the first vehicles in a queue to commence motion and enter an intersection after the traffic signal displays a green indication. Departure headway is the average time spacing in seconds between successive vehicles in an entering platoon.

\section{Data analysis and results}

\subsection{Aim, method and data analysis}

The main objective was to obtain a set of reliable and unbiased data for different queue positions at signalized intersections. It was also intended to collect as 
much information as possible so that major factors affecting start-up lost times and departure headways could be identified. Relevant information was collected for 11 signalized intersection approaches in the Kifissias Avenue, one of the major arterial roads in the Athens, Greece urban area, in late 2008. Data collection through contemporary techniques included lane width; lane position (inside lane versus outside lane, etc.); time of day (morning-peak versus afternoon-peak); and posted speed limit $(48 \mathrm{~km} / \mathrm{h}, 56 \mathrm{~km} / \mathrm{h}, 64 \mathrm{~km} / \mathrm{h}$, or $72 \mathrm{~km} / \mathrm{h})$. Statistical analyses were performed in order to define the elements of the approaches that influence the time headway values.

The manual input method was used for time increment measurements; this has an element of error because of the observer's reaction time. The error at each time headway measured was estimated. In order to accomplish that, the observers measured their experimental error. The hypothesis was that zero seconds time headway existed. Thus, the time calculated at two consecutive presses of the event button would give an estimate of the error.

This procedure was repeated numerous times and an average was calculated. The average error was found to be 0.155 seconds with standard deviation 0.014 seconds and standard error of the mean 0.0014 seconds.

As a result, all times measured in this study may contain an average measurement error of 0.155 seconds. Since this error was very small, the observer reaction time was assumed to be uniform for all inputs.

In order to edit the data set, some assumptions were made. First it was assumed that the space interval (front-axle to front-axle) between vehicles would be at least $6 \mathrm{~m}$. Second, the maximum speed that the vehicles of each position in the queue could attain, when they reach the reference line, would be as follows: $16 \mathrm{~km} / \mathrm{h}$ for the second vehicle in the queue, $24 \mathrm{~km} / \mathrm{h}$ for the third and fourth, 32 $\mathrm{km} / \mathrm{h}$ for the fifth, $40 \mathrm{~km} / \mathrm{h}$ for the sixth, $43.2 \mathrm{~km} / \mathrm{h}$ for the seventh, $48 \mathrm{~km} / \mathrm{h}$ for the eighth, $51.2 \mathrm{~km} / \mathrm{h}$ for the ninth, $54.4 \mathrm{~km} / \mathrm{h}$ for the tenth and $56 \mathrm{~km} / \mathrm{h}$ for the positions eleven through nineteen.

Upon examination of the data, some very low values for vehicle headways were discovered. Computations were performed, and some values were found to be impossible. The following editing was done for the extremely small values. Whenever values smaller than the following were found, they were interpreted as missing for the following analysis. The minimum acceptable headway for the first vehicle in the queue was $1.00 \mathrm{sec}$, as the PIJR time (Perception, Identification, Judgment and Reaction time) for an average driver in an urban area. The minimum headway values for positions two through nineteen were $1.37 \mathrm{sec}, 0.91 \mathrm{sec}, 0.91 \mathrm{sec}, 0.68 \mathrm{sec}, 0.54 \mathrm{sec}, 0.51 \mathrm{sec}, 0.45 \mathrm{sec}, 0.42 \mathrm{sec}, 0.40$ sec, and $0.39 \mathrm{sec}$ thereafter.

\subsection{Explanatory data analysis}

The analysis was concentrated only on passenger cars and light trucks (vehicles of the so-called first category). The headway times of any heavy vehicles i.e. trucks, buses and trailers (vehicles of the so-called second category), were 
excluded from the analysis. It was assumed that these vehicles influence only their headways and not of their following cars. This was examined and it was found to be valid. The headways of cars following vehicles of the second category did not have any significant difference from the cars of the same position following vehicles of the first category. Thus, the following analysis was concentrated only on passenger cars and light trucks and the results are valid only for these vehicles

This analysis is the first step to relate the headway values and their variability to physical and traffic conditions, see also Moussavi et al. [3]. For reasons discussed earlier, there is a marked tendency for the number of sample points to decrease as one goes from the front of the waiting line towards the rear. In much of the statistical analysis that follows, the shape of the density functions is important. From a practical point of view, by this it is meant that the first two moments would be reasonably accurate. For the density functions that are used in this study, it must be stressed that if the sample size were sufficiently larger to ensure a given relative accuracy of the variance, then the relative accuracy of the mean would be greater.

A normality test from the SAS/STAT software [4] was used to test the distribution of the departure headways. Departure headways were in most cases normally distributed. Departure from normality, where it existed, was in the form of positive skewing with a 10ng tail of high values

A preliminary analytical procedure involved computing selected sample statistics. Thus, the number of observations, median, mean, standard deviation, minimum, maximum, range, standard error of the mean, variance, and coefficient of variation of the vehicles' headways were computed for each specific lane that was investigated. Afterwards, the descriptive statistics for all the collected data were summarized.

The mean value of headway generally decreases from front to rear of the queue and there is some tendency to approach a constant value at position three or four, depending on the case.

An overall review of the studies in the literature review suggests that all efforts are fragmented in terms of study methods, location characteristics, and technical objectives. Therefore, comparison of these studies is limited to a general observation that their results do not entirely agree. It is also noticed that by far the most comprehensive study was that reported by Greenshields et al. conducted 60 years ago (Greenshields [5]); there is no doubt that it is questionable whether the results could represent current traffic characteristics. In addition, the limited depth of the studies does not allow a clear identification of factors that can affect the headway values

It is interesting to note that all the entering headways from various studies appear to follow a similar pattern except for the first two vehicles. It is a fact that studies with the first vehicle having a low headway value have used a different definition of entering headway than the others. This study defines the reference line as an imaginary line in front of the usual first vehicles stopping place. So, the departure headway for the first vehicle is closer to its PIJR time. 


\section{Equation development}

The intention was to develop an equation which would relate the time required for a number of stopped vehicles at a signalized intersection to pass the reference line with the start-up lost time, $L$, and the time headway, $H$, between vehicles. The equation can have the following form.

$$
\mathrm{G}=\mathrm{L}+\mathrm{H}^{*} \mathrm{n} \text { for } \mathrm{n} \geq \mathrm{a}
$$

where $G=\quad$ time needed for the front of $\eta$ vehicles in a single-line stopped queue to cross a designated reference line at a signalized intersection after the signal indication changes to green

$\mathrm{L}=\quad$ start-up lost time

$\mathrm{H}=$ average time headway between successive vehicles

$\mathrm{n}=$ number of vehicles that cross the reference line

$\mathrm{a}=$ the number of vehicles that contribute to the start-up lost time

From the field observations and the analyses that followed the variable $\alpha$ could be determined. On the assumption that vehicle headways remain constant after the front of the $\alpha^{\text {th }}$ vehicle had crossed the reference line, the start-up lost time was estimated. In order to estimate the variable $H$, the mean values for the positions from $\alpha$ to the end of the queue were calculated. The variable $L$ was the difference of the means of the $\alpha$ positions and the average time headway for the remaining vehicles, $H$.

The data analysis showed that the start-up lost time varied from 0.35 to 1.39 seconds. The constant value of headway that was approached by vehicles, after the ones that contributed to the start-up lost time, ranged from 1.76 to 1.94 seconds. When all data were analyzed together, the overall average start-up lost time of 1.34 seconds can be attributed to the first four vehicles and the average headway, $\mathrm{H}$, after the fourth vehicle was 1.82 seconds.

The start-up lost times observed in this study are considerably smaller than the ones reported in the literature. Differences can be expected in start-up lost times when different screen line definitions are used. In this study, the elapsed time from the beginning of the green interval includes reaction (PIJR) time, but no acceleration time.

\section{Factors affecting the start-up lost times and departure headways}

The need for reliable and readily obtainable estimates of vehicle headways has generated a considerable amount of research devoted to the determination of the factors affecting vehicle headways. Table 1 summarizes the various elements typically considered within each of the factors.

In the present study, the first step was to test the hypothesis that all mean start-up lost times and headways for each lane were equal. The results are shown in Table 2. The hypothesis can be rejected at the 0.001 significance level, which means that all mean start-up lost times and headways in each lane are not equa1. Note that the minimum value that the statistical software used could report was 0.0001 . 
The second part of the conducted analysis, in the study reported herein, focused on finding out whether selected factors were influencing start-up lost times and vehicle headways. Because the variety of existing conditions was limited at the intersections observed, the factors that could be examined included only lane width, lane position, time of day and posted speed limit. Most of these factors could not be expressed in continuous terms, therefore factor analysis or regression analysis could not be applied. Consequently, a simple comparative approach was engaged.

In this approach, the factors were analyzed one at a time. Limiting values of all but one important variable allowing that variable to vary performed analysis, and observing the effect of that variable on the start-up lost time and the headways.

For the investigation of the lane width factor, it was necessary to group the studied lanes into three groups, in order to have a more dependable number of observations within each group. The groups were the following:

1. $\quad$ Lane width less than $3.2 \mathrm{~m}$.

2. Lane width between $3.2 \mathrm{~m}$ and $3.6 \mathrm{~m}$

3. Lane width more than $3.6 \mathrm{~m}$

Table 1: $\quad$ Summary of principal factors affecting headways at signalized intersections.

\begin{tabular}{ll}
\hline Factors & Elements affecting vehicle headways \\
\hline Geometrics & Width of approach \\
Width of lanes & Number of lanes \\
& Grade \\
& Radius of turns \\
& Length of turn bay \\
Operating conditions & Signal timing and phasing arrangements \\
& Peaking characteristics \\
& Parking activities \\
& Bus stop operations \\
Traffic characteristics & Traffic composition \\
& Turning movements \\
& Pedestrian activity \\
Environmental and other factors & Weather \\
& Driver behavior \\
Area population \\
Roadway surface conditions \\
Adjacent land uses
\end{tabular}


Table 2: $\quad$ Analysis of variance for start-up lost times and headways.

\begin{tabular}{llrrrrr}
\hline & Source & DF & SS & Mean Square & F value & Pr $>F^{*}$ \\
\hline $\begin{array}{l}\text { Start-up } \\
\text { lost time }\end{array}$ & Model & 15 & 20.871632 & 1.391442 & 5.40 & 0.0001 \\
& Error & 1576 & 406.241520 & 0.257767 & & \\
& Corrected Total & 1591 & 427.113152 & & & \\
& Model & 1 & 19.409348 & 1.293957 & 6.96 & 0.0001 \\
& & 5914 & 1096.744632 & 0.185889 & & \\
Headway & Error & & & & & \\
& & Corrected Total 5915 & 1116.153980 & & & \\
\hline
\end{tabular}

The analysis for the lane position effect consisted from four different groups. The lane position was measured from the centerline of the investigated approach (i.e. lane position 1 indicates that this is the lane closest to the centerline, usually called inside lane). The four groups were for lane positions 1, 2, 3, or 4 .

The effect of the time of the day was examined through comparisons for a.m. peak periods and p.m. peak periods.

The data was collected at areas with posted speed limit of $48 \mathrm{~km} / \mathrm{h}, 56 \mathrm{~km} / \mathrm{h}$, $64 \mathrm{~km} / \mathrm{h}$, and $72 \mathrm{~km} / \mathrm{h}$. The effect of the speed limit was investigated for the four distinct groups.

Again, analysis of variance (ANOVA) was performed for unbalanced data (Hinkelmann [6]).The outcome of the analysis on the effect of speed limit on start-up lost times and departing headways is that the start-up lost times are not significantly different for business area with $48 \mathrm{~km} / \mathrm{h}$ speed limit, intermediate area with $56 \mathrm{~km} / \mathrm{h}$ speed limit, and commercial area with $64 \mathrm{~km} / \mathrm{h}$ speed limit; and the vehicle headways are not significantly different for intermediate area with $35 \mathrm{mph}$ speed limit, commercial area with $56 \mathrm{~km} / \mathrm{h}$ speed limit, and open area with $72 \mathrm{~km} / \mathrm{h}$ speed limit.

Because of the limited variety of sites studied, the individual impacts of the factors could not be isolated by using statistical methods. Therefore, results would be exaggerated if a particular group contained a large amount of collected data.

In a relevant study by Shawaly et al. [7], the results showed a considerable variation in the departure patterns of vehicles crossing the stop line during the peak hours not only during a particular peak period, but also on different dates.

Recent models use a log-normal distribution model for departure headways (Xuexiang Jin [8]). 


\section{Summary of findings}

Let summarize the findings:

(i)The mean value of headway generally decreases from front to rear of the queue.

(ii)The hypothesis of equal means was rejected at the 0.001 level of significance, and the effect of position within the queue on mean headways between pairs of vehicles is thus found to be significant. The general observation is that the startup lost time of a line of stopped vehicles can best be attributed to the reaction time and starting performance of the first 2 or 3 vehicles in line, depending on the case. This result is within the range of values reported in the literature.

(iii)The start-up lost times ranged from 0.35 to 1.39 seconds and were attributed to the first two or three vehicles.

(iv)The vehicle headways, which varied from 1.76 to 1.94 seconds, approached a constant value after these first vehicles.

(v)When all data were analyzed together, the overall average start-up lost time of 1.34 seconds can be attributed to the first four vehicles and the average headway after the fourth vehicle was 1.82 seconds.

(vi)The hypothesis that all mean start-up lost times were equal was tested. The hypothesis was rejected at the 0.001 significance level. The hypothesis that all mean vehicle headways were equal was rejected at the $0.0011 \mathrm{evel}$ of significance.

(vii)The factors that affect the start-up lost times and the vehicle headways were investigated. It was found that the lane width (which varied from 2.85 to $5.4 \mathrm{~m}$ ) and the lane position (for lane positions 1 through 4 ) did not have a significant effect on either the start-up lost time or the vehicle headways. On the other hand, the combined effect of time of day and direction had a significant effect on both the start up lost time and the vehicle headways. The combined effect of posted speed limit and area had a significant effect for start-up lost time when the speed limit was $45 \mathrm{mph}$ in an open area and for vehicle headways when the speed limit was $30 \mathrm{mph}$ in a business area.

\section{Conclusions}

As the problems of efficiently transporting people and goods in modern urban areas persist and intensify, there is accentuated concern for provision and improved operation of systems of both individual and mass transport facilities. Emphasis has concentrated on mass transit. Nevertheless, surface arterial streets and highways are paramount, as they continue to bear the brunt of enormous traffic demands. Literally hundreds of billions of vehicle-kms are traveled annually on city streets.

In street and highway systems incorporating signalized intersections, the intersection has always been the cardinal element. To improve the operational effectiveness of the system, it is necessary to develop traffic signalization to its highest possible level of efficiency. 
The number of waiting vehicles that can cross a signalized street intersection in a given time depends in the simple case on how soon they move after the signal changes to the green indication and on how fast they accelerate. The driver of the first vehicle reacts to the signal change or the clearance of the intersection and then each driver in turn reacts until the ripple of motion has traveled to the tail car of the queue, with the progress of the wave of motion depending on individual reaction times. It is the total time required to pass a given number of vehicles through the intersection that is of primary interest to the traffic engineer. In analytical categories, this time depends on the integration of individual patterns of reaction times, acceleration, speed and spacing.

The overall review of the studies found in the literature indicated that there is a high degree of variability among the start-up lost times and departure headways at signalized intersections. Most studies were based on limited data points and they date back 10 to 30 years ago. Since the traffic and vehicle characteristics have changed over time, the validity of the results of these studies -that do not reflect the current traffic and vehicle characteristic- is questionable. Therefore, there is a need to reexamine the departure headways at signalized intersections. The new departure headways should also be tested for their applicability and transferability to other locations.

\section{References}

[1] A policy on geometric design of highways and streets. American Association of State Highway and Transportation Officials, Washington, D.C., 1990.

[2] Stokes, Robert W., Comparison of saturation flow rates at signalized intersections. ITE journal, Institute of Transportation Engineers, Vol. 58, No. 11, November 1988, pp. 15-20.

[3] Moussavi, Massoum and Tarawneh, Mohammed, Variability of departure headways at signalized intersections. ITE 2004 Compendium of Technical Papers, Institute of Transportation Engineers, 2004.

[4] SAS Institute Inc., SAS/STAT Release 6.03 Edition. Cary, NC: SAS Institute Inc: 2003.

[5] Greenshields, Bruce D., Schapiro, Donald and Ericksen, Elroy, Traffic performance at urban street intersections. Technical Report 1, Yale Bureau of Highway Traffic, Eno Foundation for Highway Traffic Control, 1947.

[6] Hinkelmann, Klaus and Kempthorne, Oscar , Design and Analysis of Experiments. Volume I: Introduction to Experimental Design (Second ed.). Wiley, 2008 (Ref. ANOVA)

[7] Shawaly, E.A.A., Ashworth, R., and Laurence, C.J.D., A comparison of observed, estimated and simulated queue lengths and delays at oversaturated signalized junctions. Traffic Engineering + Control, Vol. 29, No. 12, England, December 1998, pp. 637-643.

[8] Xuexiang Jin, Yi Zhang, Fa Wang, Li Li, Danya Yao, Yuelong Su and Zheng Wei , Departure headways at signalized intersections: A log-normal distribution model approach. Transportation Research Part C: Emerging Technologies, Volume 17, Issue 3, June 2009, Pages 318-327 